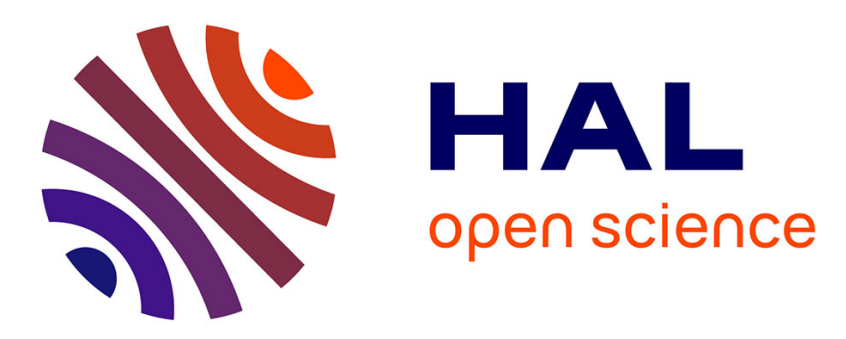

\title{
Dual-Polarized Generalized Spatial Modulation for Wireless Terabit System
}

\author{
Nizar Bouhlel, Majed Saad, Faouzi Bader, Jacques Palicot
}

\section{To cite this version:}

Nizar Bouhlel, Majed Saad, Faouzi Bader, Jacques Palicot. Dual-Polarized Generalized Spatial Modulation for Wireless Terabit System. 31st IEEE International Symposium on Personal, Indoor and Mobile Radio Communications (PIMRC'2020), Aug 2020, London, United Kingdom. hal-02876509

\section{HAL Id: hal-02876509 \\ https://hal.science/hal-02876509}

Submitted on 20 Jun 2020

HAL is a multi-disciplinary open access archive for the deposit and dissemination of scientific research documents, whether they are published or not. The documents may come from teaching and research institutions in France or abroad, or from public or private research centers.
L'archive ouverte pluridisciplinaire HAL, est destinée au dépôt et à la diffusion de documents scientifiques de niveau recherche, publiés ou non, émanant des établissements d'enseignement et de recherche français ou étrangers, des laboratoires publics ou privés. 


\title{
Dual-Polarized Generalized Spatial Modulation for Wireless Terabit System
}

\author{
Nizar Bouhlel ${ }^{1}$, Majed Saad ${ }^{1}$, Faouzi Bader ${ }^{1,2}$ and Jacques Palicot ${ }^{1}$. \\ ${ }^{1}$ IETR/CentraleSupélec, Rennes Campus, Cesson-Sévigné-France \\ ${ }^{2}$ ISEP- Institut Supérieur d'Électronique de Paris. \\ \{nizar.bouhlel, majed.saad, jacques.palicot\}@centralesupelec.fr, and faouzi.bader@isep.fr
}

\begin{abstract}
In this paper, we address multiple-input multipleoutput (MIMO) communication technique based on generalized spatial modulation (GSM) and dual-polarized (DP) antennas in sub-TeraHertz (sub-THz) bands to improve the Spectral Efficiency (SE) and to reduce the spatial correlation effect, and the space occupancy. The proposed system adds the polarization dimension to the conventional GSM scheme and enhance the SE. Moreover, the joint Maximum Likelihood (ML) detector and a Modified Ordered Block Minimum Mean-Squared Error (MOBMMSE) detector algorithm is proposed to detect jointly the complex symbols and the virtual bits conveyed by the activated polarization and the transmit antenna combination index. MOBMMSE can achieve near-ML performance with low complexity. The performance of DP-GSM system over (un)correlated Rayleigh/Rician fading and sub-THz channels has been studied and the results show that DP-GSM system is robust against channel deficiency such as Rician fading and spatial correlation. Moreover, the Dual polarized Spatial Multiplexing (DP-SMX) and the Uni-Polarized GSM (UP-GSM) are compared with the DP-GSM system. The results reveal that DP-GSM with low order modulation schemes as QPSK outperforms the DP-SMX at high spatial correlations, and thus it is a promising candidate for ultra-high wireless data rates communication in Sub-THz band.

Index Terms-Generalized spatial modulation (GSM), Dual polarization (DP), Beyond 5G, Multiple-input multiple-output (MIMO), Index Modulation (IM), Sub-Terahertz (sub-THz), Spectral efficiency, Spatial Multiplexing (SMX).
\end{abstract}

\section{INTRODUCTION}

Multiple-input multiple-output (MIMO) techniques have been widely used during the last two decades and have been integrated into most of the recent digital wireless communication standards [1], because of their advantages in terms of diversity/multiplexing gain [2]. However, in order to meet the ever-increasing demands in terms of increased capacity, high data throughput and better reliability, MIMO still needs improved transceivers to be designed. To reach these goals, wireless ultra-high data rate needs a high system Spectral Efficiency (SE) and a large bandwidth available in the millimeter wave $(\mathrm{mmW})$ and sub-TeraHertz (sub-THz) bands, that are considered for beyond 5G ultra-high data rate scenarios such as the close proximity peer-to-peer (P2P) applications (Kiosk downloading scenario), intra-device communication, wireless fronthaul/backhaul and data centers [3].

In the context of BRAVE project [4], we explore new techniques for wireless terabit communication system. Thus, Index Modulation (IM) with advanced MIMO schemes and a huge bandwidth (around $50 \mathrm{GHz}$ ) that can be allocated in the sub-
THz bands (mainly between $90 \mathrm{GHz}-200 \mathrm{GHz}$ ) [5], are the key enablers to increase the SE and data rates. Spatial modulation (SM) has been recognized as a low-complexity MIMO scheme [6], where a single antenna at each time slot is activated to convey information by its index. The main advantage of SM is that the interference at the receiver and the inter-antenna synchronization at transmitter are avoided. However, the SE of the SM-MIMO technique increases logarithmically with number of transmit antennas (TAs).

To further increase the SE, the Uni-Polarized Generalized Spatial Modulation (UP-GSM) technique was developed in [7]. It uses the space dimension of antenna array to conveys information by the index of the activated transmit antenna combination (TAC) and by the M-ary symbols [8]. In [9], it was shown that using the UP-GSM with power efficient single carrier modulations can successfully achieve a low power Terabits system.

Despite the multiple advantages of UP-GSM system, the latter suffers from an important performance degradation with Rician channel and in spatially correlated channels. Note that the degradation due to spatial correlation is reduced in [10] but cannot be completely eliminated. In addition, the space occupancy of uni-polarized (UP) antenna arrays in MIMO systems can limit the number of antennas due to physical space limitation especially at low frequency bands. To deal with the space limitation, an efficient solution has been proposed for the SM-MIMO system, by adding a polarization dimension to the transmitter and receiver using dual-polarized (DP) antennas that can transmit simultaneously through two orthogonal polarization directions. But in DP-SM [11] only one polarization is used to allow better separation between channels and thus better performance in highly correlated channel. At the receiver, the detection algorithm for UP-GSM involves the estimation of both the transmitted M-ary symbols and the index of the activated TAC that can be performed jointly using maximum-likelihood (ML) detector or using suboptimal detectors as the ordered blocked minimum meansquared error (OB-MMSE) [12].

In this paper, we propose a DP-GSM scheme where we incorporate DP antennas with GSM to reduce the spatial correlation impact and also to enhance the system SE by exploring the polarization dimension. In addition, DP-GSM allows to reduce the space occupancy of antenna arrays by half without any performance degradation compared to UP- 


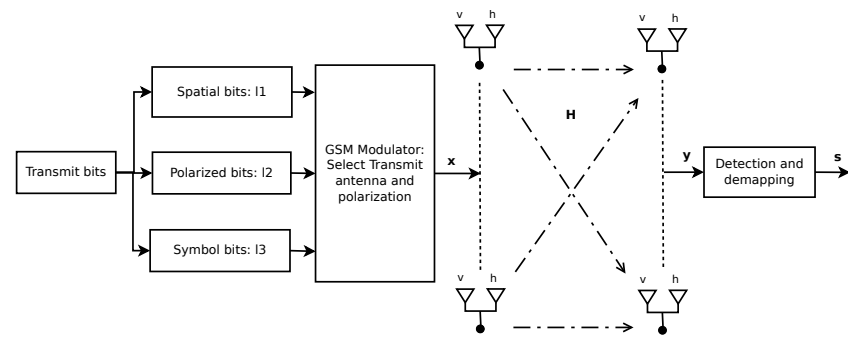

Fig. 1. $N_{r} \times N_{t}$ DP-GSM system model.

GSM. In contrast to UP-GSM, DP-GSM should detect the polarization bits conveyed by the activated polarization of each active antenna, in addition to the estimation of the spatial and the symbol bits. For this reason, the joint maximumlikelihood (ML) detector for DP-GSM that achieves optimal performance is proposed, and a sub-optimal detector of lower complexity, named Modified OB-MMSE (MOB-MMSE), is presented to additionally estimate the polarization bits compared to OB-MMSE. Moreover, the performance analysis of the proposed DP-GSM system is addressed over spatially correlated/uncorrelated Rayleigh Rician channels with different cross-polarization correlation, and also over sub- $\mathrm{THz}$ channels. To further illustrate the potential of the DP-GSM system, the UP-GSM and the dual polarized multiple-input multiple-output systems with Spatial Multiplexing (DP-SMX) are compared against our system.

The paper is organized as follows. In section II, we describe the DP-GSM system. Section III illustrates the channel modeling with spatial and polarization correlation whereas the performance analysis is presented in section IV. Finally, the discussion, and some concluding remarks close up this paper.

\section{System MOdeL: DUAL-Polarized GSM SYSTEM}

The proposed system model is a MIMO system with $N_{t}$ and $N_{r}$ DP antennas at transmitter and receiver, respectively, and based on the GSM, in which $N_{a}\left(N_{a}<N_{t}\right)$ antennas are activated in each time slot. Therefore, the number of TAC combinations is $C_{N_{t}}^{N_{a}}$ that represents the binomial coefficient. However, only $2^{\left\lfloor\log _{2}\left(C_{N_{t}}^{N a}\right)\right\rfloor}$ antenna combinations are permitted to keep the bits length an integer number, where $\lfloor x\rfloor$ is the greatest integer smaller than $x$.

The $N_{r} \times N_{t}$ DP-GSM system leads to $2 N_{r} \times 2 N_{t}$ dimensional channel between transmitter and receiver due to the implemented dual polarization. The DP-GSM system is shown in Fig. 1. At each transmission instant, a block of $L=$ $L_{1}+L_{2}+L_{3}$ incoming data bits are selected and constituted of three parts named: $L_{1}=\left\lfloor\log _{2}\left(C_{N_{t}}^{N_{a}}\right)\right\rfloor$ bits are used to select the $N_{a}$ activated antennas at the transmitter, $L_{2}=N_{a} \log _{2} N_{p}$ bits used to select the polarizations of the selected antennas. Here $N_{p}=2$ stands the polarization dimension. Note that the polarization directions can be vertical, denoted by ' $v$ ' or horizontal denoted ' $h$ '. The remaining $L_{3}=N_{a} \log _{2} M$ represent the bits mapped into $N_{a} M$-ary symbol of the transmitted constellation symbol vector $\mathbf{s}=\left[s_{1}, s_{2}, \ldots, s_{N_{a}}\right]^{\mathrm{T}}$ where $[.]^{\mathrm{T}}$ is the transpose operator. Thus, the total achieved $\mathrm{SE}$ in bit per channel use (bpcu) can be expressed as

$$
L=\left\lfloor\log _{2}\left(C_{N_{t}}^{N_{a}}\right)\right\rfloor+N_{a} \log _{2} M+N_{a}
$$

By doing so, the number of possible TACs is $N_{1}=$ $2^{\left\lfloor\log _{2}\left(C_{N_{t}}^{N a}\right)\right\rfloor}$ and the number of possible transmit polarization combinations (TPCs) is $N_{2}=2^{N a}$. Thus the total number of possible combinations using TACs and TPCs is $N=N_{1} N_{2}$.

The transmitted signal is denoted by $\mathbf{x}$ and represented by a $2 N_{t} \times 1$ vector whose entries have $N_{a}$ non-zero elements. The position of the non-zero elements in $\mathbf{x}$ allows the identification of the selected TAs as well as their polarizations and the transmitted symbols. We denote in this work $i_{1}, i_{2}, \ldots$, $i_{N_{a}}$ as the indices of $N_{a}$ active antenna in the $i$-th TAC, where $i \in\left\{1,2, \ldots, N_{1}\right\}$ and we denote $\ell_{1}, \ell_{2}, \ldots, \ell_{N_{a}}$ as the corresponding polarization of the activated antennas at the $\ell$-th TPC where $\ell \in\left\{1,2, \ldots, N_{2}\right\}$. The TA with index $i_{q} \in\left\{1,2, \ldots, N_{t}\right\}$ and polarization $\ell_{q} \in\{\mathrm{v}, \mathrm{h}\}$ sends out symbols $s_{q}$ where $q \in\left\{1,2, \ldots, N_{a}\right\}$. The transmit signal vector $\mathbf{x}$ is given by

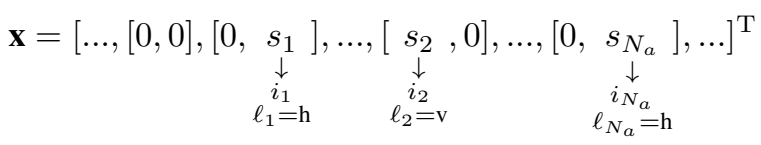

At receiver, $N_{r}$ DP antennas receive the modulated signal transmitted over a slow fading and frequency-flat MIMO channel. The channel matrix $\mathbf{H}$ is equivalent to a $2 N_{r} \times 2 N_{t}$ matrix represented as

$$
\mathbf{H}=\left[\begin{array}{ccccc}
h_{1 \mathrm{v}, 1 \mathrm{v}} & h_{1 \mathrm{v}, 1 \mathrm{~h}} & h_{1 \mathrm{v}, 2 \mathrm{v}} & \cdots & h_{1 \mathrm{v}, N_{t} \mathrm{~h}} \\
h_{1 \mathrm{~h}, 1 \mathrm{v}} & h_{1 \mathrm{~h}, 1 \mathrm{~h}} & h_{1 \mathrm{~h}, 2 \mathrm{v}} & \cdots & h_{1 \mathrm{~h}, N_{t} \mathrm{~h}} \\
h_{2 \mathrm{v}, 1 \mathrm{v}} & h_{2 \mathrm{v}, 1 \mathrm{~h}} & h_{2 \mathrm{v}, 2 \mathrm{v}} & \cdots & h_{2 \mathrm{v}, N_{t} \mathrm{~h}} \\
\vdots & \vdots & \vdots & \vdots & \vdots \\
h_{N_{r} \mathrm{~h}, 1 \mathrm{v}} & h_{N_{r} \mathrm{~h}, 1 \mathrm{~h}} & h_{N_{r} \mathrm{~h}, 2 \mathrm{v}} & \cdots & h_{N_{r} \mathrm{~h}, N_{t} \mathrm{~h}}
\end{array}\right] \text {, }
$$

where each $h_{i^{\prime} u, j^{\prime} u^{\prime}}$ and $u, u^{\prime} \in\{\mathrm{v}, \mathrm{h}\}$, represents the fading coefficient between polarization $u$ of $i^{\prime}$-th receive antenna and the polarization $u^{\prime}$ of $j^{\prime}$-th TA. If $u^{\prime}=u, h_{i^{\prime} u, j^{\prime} u^{\prime}}$ represents the co-polar channel coefficient; otherwise, it represents the cross-polar channel coefficient. We can reformulate (3) as $\mathbf{H}=\left[\mathbf{H}_{1}, \mathbf{H}_{2}, \ldots, \mathbf{H}_{N_{t}}\right]$ where $\mathbf{H}_{j^{\prime}}=\left[\mathbf{h}_{j^{\prime} \mathrm{v}}, \mathbf{h}_{j^{\prime} \mathbf{h}}\right] \in \mathbb{C}^{2 N_{r} \times 2}$ and $\mathbf{h}_{j^{\prime} \mathrm{v}}=\left[h_{1 \mathrm{v}, j^{\prime} \mathrm{v}}, h_{1 \mathrm{~h}, j^{\prime} \mathrm{v}}, h_{2 \mathrm{v}, j^{\prime} \mathrm{v}}, \ldots, h_{N_{r} \mathrm{~h}, j^{\prime} \mathrm{v}}\right]^{\mathrm{T}}$ is the a $2 N_{r} \times 1$ channel matrix for polarization $\mathrm{v}$ of $j^{\prime}$-th TA. Similarly, $\mathbf{h}_{j^{\prime} \mathrm{h}}=\left[h_{1 \mathrm{v}, j^{\prime} \mathrm{h}}, h_{1 \mathrm{~h}, j^{\prime} \mathrm{h}}, h_{2 \mathrm{v}, j^{\prime} \mathrm{h}}, \ldots, h_{N_{r} \mathrm{~h}, j^{\prime} \mathrm{h}}\right]^{\mathrm{T}}$ is the a $2 N_{r} \times 1$ channel matrix for polarization $\mathrm{h}$ of the $j^{\prime}$-th TA. To be able to model correctly the channel $\mathbf{H}$, two kind of polarizations are investigated in our paper. Correlations induced by polarization effects within each antenna, and the limited spacing between antennas [11]. These effects will be studied in the next section. The received signal $\mathbf{y} \in \mathbb{C}^{2 N_{r} \times 1}$ can be formulated as

$$
\mathbf{y}=\mathbf{H} \mathbf{x}+\mathbf{n}=\sum_{q=1}^{N_{a}} \mathbf{h}_{i_{q} \ell_{q}} s_{q}+\mathbf{n}=\mathbf{H}_{I, P} \mathbf{s}+\mathbf{n}
$$

where $\mathbf{n} \in \mathbb{C}^{2 N_{r} \times 1}$ is additive white complex Gaussian noise with zero mean and variance $\sigma^{2}, \mathbf{H}_{I, P}=$ 
$\left[\mathbf{h}_{i_{1} \ell_{1}}, \mathbf{h}_{i_{2} \ell_{2}}, \ldots, \mathbf{h}_{i_{N_{a}} \ell_{N_{a}}}\right]$ is the sub-matrix with $N_{a}$ columns of $\mathbf{H}$, corresponding to the TAC set $I$ and TPC set $P$.

A. Joint-ML Detector

The receiver estimates the activated antennas, their polarizations and the transmitted symbols by using joint ML detection which performs exhaustive search over all the possible transmitted signal vectors and is given by

$$
\hat{\mathbf{x}}=\underset{\mathbf{x} \in \chi}{\arg \min }\|\mathbf{y}-\mathbf{H x}\|^{2}
$$

where $\chi$ denotes the set of all possible transmit vectors in spatial, polarization and signal constellation domains, and $\hat{\mathbf{x}}$ is the estimated transmit vector. Based on (4), $\hat{\mathbf{x}}$ can be written as

$$
(\hat{I}, \hat{P}, \hat{\mathbf{s}})=\underset{I \in \mathbb{I}, P \in \mathbb{P}, \mathbf{s} \in \mathbb{Q}}{\arg \min }\left\|\mathbf{y}-\mathbf{H}_{I, P} \mathbf{S}\right\|^{2}
$$

where $\mathbb{I}=\left\{I_{1}, I_{2}, \ldots, I_{N_{1}}\right\}, I_{i}$ is defined as the set of the $N_{a}$ active TAs in the $i$-th TAC, $\mathbb{P}=\left\{P_{1}, P_{2}, \ldots, P_{N_{2}}\right\}, P_{\ell}$ is defined as the set of the $N_{a}$ active polarization in the $\ell$-th TPC, and $\mathbb{Q}$ is the set of $N_{a}$-dimensional modulation symbol vectors.

\section{B. Modified OB-MMSE Detector}

In order to reduce complexity by avoiding the use of the joint ML detector, we propose a modified version of OBMMSE detector which has been successfully used for UPGSM where polarization dimension is not used [12]. Therefore, in addition to the estimation of the indices of the $N_{a}$ possible activated TAs, we propose to estimate their polarizations. Concretely, the pseudo-inverse of channel column $z_{i_{q} \ell_{q}}$ is computed and then weighting factors $w^{i, \ell}$ are calculated to measure the joint reliability of (TAC, TPC) as follows:

$$
w^{i, \ell}=\sum_{q=1}^{N_{a}} z_{i_{q} \ell_{q}}^{2} \quad \text { with } z_{i_{q} \ell_{q}}=\frac{\mathbf{h}_{i_{q} \ell_{q}}^{H} \mathbf{y}}{\mathbf{h}_{i_{q} \ell_{q}}^{H} \mathbf{h}_{i_{q} \ell_{q}}}
$$

We recall the reader that the total number of TA and polarization combination $(i, \ell)$ corresponds to $N$. Sorting the weighing factor values of the vector $\mathbf{w}=\left[w^{i, \ell} ; i \in\left\{1,2, \ldots, N_{1}\right\}, \ell \in\right.$ $\left.\left\{1,2, \ldots, N_{2}\right\}\right]^{\mathrm{T}}$ in descending order, we obtain the ordered activated TA and polarization combination (TAPC) as follows

$$
\left[k_{1}, k_{2}, \ldots, k_{N}\right]=\arg \operatorname{sort}(\mathbf{w})
$$

Note that the TAPC index $k_{n}$ contains implicitly the TAC and TPC indices $(i, \ell)$ and it is represented by $I_{i \leftarrow k_{n}} P_{\ell \leftarrow k_{n}}$. Consequently, a block MMSE detector with dimension of $2 N_{r} \times N_{a}$ is used for each possible TAPC to detect the vector symbol $\tilde{\mathbf{s}}_{n}$ with $n \in\{1,2, \ldots, N\}$

$\tilde{\mathbf{s}}_{n}=D\left(\left(\mathbf{H}_{I_{i \leftarrow k_{n}}^{H} P_{\ell \leftarrow k_{n}}} \mathbf{H}_{I_{i \leftarrow k_{n}} P_{\ell \leftarrow k_{n}}}+\sigma^{2} \mathbf{I}\right)^{-1} \mathbf{H}_{I_{i \leftarrow k_{n}} P_{\ell \leftarrow k_{n}}} \mathbf{y}\right)$

where $\mathbf{I}$ is the $N_{a} \times N_{a}$ identity matrix and $D($.$) is the$ digital demodulator function. For the reduction of the detection complexity of all $N$ possible TAPC, the MOB-MMSE will end once the output $\left(i, \ell, \tilde{\mathbf{s}}_{n}\right)$ satisfies the following relation (see the process to line 10 - Algorithm 1)

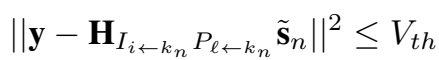

where $V_{t h}=2 N_{r} \sigma^{2}$ is a threshold to be set to produce the detected signal vector. If the $n$-th output satisfies the condition (10), the detector will provide the optimal TAC $\hat{I}$ and TPC $\hat{P}$, and the estimated symbol vector $\tilde{\mathbf{s}}_{n}$. Otherwise, the detector will continue the same procedure with $n=n+1$ until $n>N$. In this case, the detector becomes equivalent to the optimal ML algorithm as follows (See steps (14-17) in the Algorithm 1)

$$
\left\{\begin{array}{l}
u=\underset{n}{\arg \min } d_{n}, n \in\{1,2, \ldots, N\} \\
\hat{I}=I_{i \leftarrow k_{u}}, \hat{P}=P_{\ell \leftarrow k_{u}}, \hat{\mathbf{s}}=\tilde{\mathbf{s}}_{u} .
\end{array}\right.
$$

The MOB-MMSE detection algorithm is summarized in Algorithm 1.

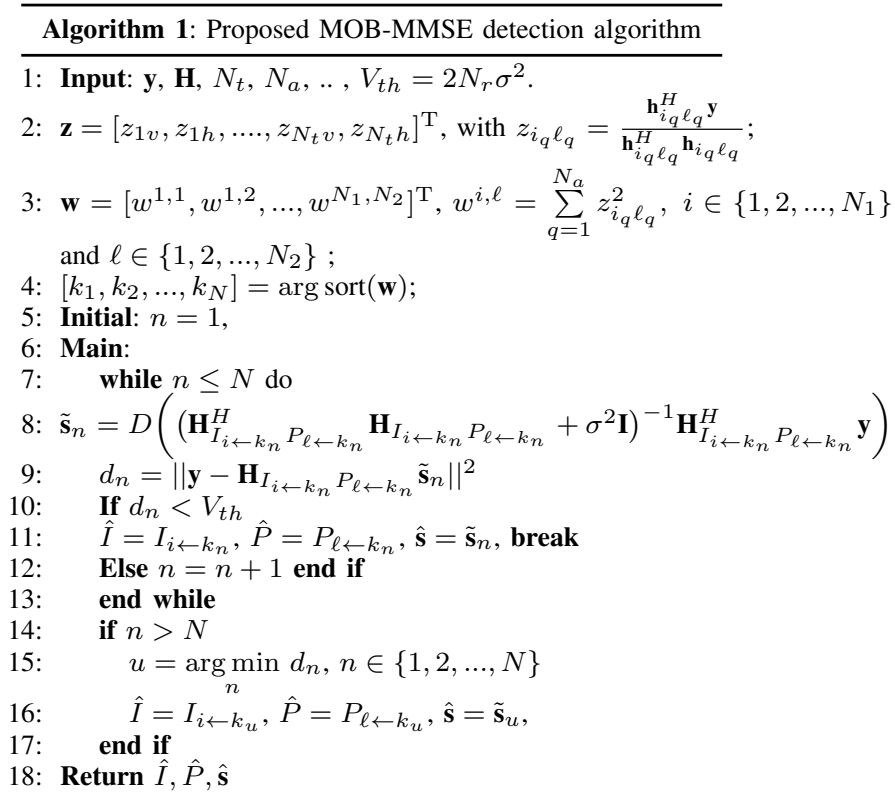

\section{CHANNEL MODEL}

The DP-GSM system may experience spatial correlation at transmitter and receiver due to the insufficient spacing among antennas and can be modeled as a matrix form, $\boldsymbol{\Sigma}_{t}$ and $\boldsymbol{\Sigma}_{r}$, respectively. Various models are used to describe the spatial correlations, and the exponential correlation model is used in this study [13]. Therefore, the correlation among $i^{\prime}$-th and $j^{\prime}$-th antenna is affected by the correlation factor $|\beta|<1$ : $\left|\boldsymbol{\Sigma}_{t}\right|_{i^{\prime}, j^{\prime}}=\beta^{\mid i^{\prime}-j^{\prime}} \mid$

The DP-GSM system may suffer also from the cross polarization correlation (XPC) between orthogonal polarization directions as shown in [14]. The polarization correlation at the transmitter and the receiver can be expressed as follows

$$
\boldsymbol{\Pi}_{t}=\left[\begin{array}{cc}
1 & \gamma_{t} \\
\gamma_{t}^{*} & 1
\end{array}\right], \quad \boldsymbol{\Pi}_{r}=\left[\begin{array}{cc}
1 & \gamma_{r} \\
\gamma_{r}^{*} & 1
\end{array}\right],
$$

respectively, with

$$
\begin{gathered}
\gamma_{t}=\frac{E\left\{h_{i^{\prime} \mathrm{v}, i^{\prime} \mathrm{v}} h_{i^{\prime} \mathrm{v}, i^{\prime} \mathrm{h}}^{*}\right\}}{\sqrt{\mu(1-\mu)}}=\frac{E\left\{h_{i^{\prime} \mathrm{h}, i^{\prime} \mathrm{v}} h_{i^{\prime} \mathrm{h}, i^{\prime} \mathrm{h}}^{*}\right\}}{\sqrt{\mu(1-\mu)}} \\
\gamma_{r}=\frac{E\left\{h_{j^{\prime} \mathrm{v}, j^{\prime} \mathrm{v}} h_{j^{\prime} \mathrm{h}, j^{\prime} \mathrm{v}}^{*}\right\}}{\sqrt{\mu(1-\mu)}}=\frac{E\left\{h_{j^{\prime} \mathrm{v}, j^{\prime} \mathrm{h}} h_{j^{\prime} \mathrm{h}, j^{\prime} \mathrm{h}}^{*}\right\}}{\sqrt{\mu(1-\mu)}}
\end{gathered}
$$

where the parameter $0<\mu \leq 1$ is the amount of power leakage from one polarization to the other [15]. Assuming 
the symmetry condition between different polarization, the leakage parameter is given as follows $\mu=E\left\{\left|h_{i^{\prime} \mathrm{v}, i^{\prime} \mathrm{h}}\right|^{2}\right\}=$ $E\left\{\left|h_{i^{\prime} \mathrm{h}, i^{\prime} \mathrm{v}}\right|^{2}\right\}$ and $1-\mu=E\left\{\left|h_{i^{\prime} \mathrm{v}, i^{\prime} \mathrm{v}}\right|^{2}\right\}=E\left\{\left|h_{i^{\prime} \mathrm{h}, i^{\prime} \mathrm{h}}\right|^{2}\right\}$. In the literature, the cross-polarization discrimination (XPD) is defined as the ratio between $1-\mu$ and $\mu$. For larger XPD values, the two polarization directions are easier to be discriminated. In [16], it has been demonstrated that the impact of the cross-polarization is relatively small and $0 \leq\left|\gamma_{r}\right|,\left|\gamma_{t}\right| \leq 0.2$.

In our study, we consider a slow fading MIMO channel matrix as Rayleigh or Rician multipath fading channel with/without spatial correlation and with polarization correlation. Therefore, the $2 N_{r} \times 2 N_{t}$ DP-polarized channel matrix is expressed in the Kronecker form given by

$$
\begin{array}{r}
\mathbf{H}=\sqrt{\frac{K}{1+K}}\left(\mathbf{1}_{N_{r} \times N_{t}} \otimes \boldsymbol{\Gamma}\right) \odot \mathbf{H}_{\mathrm{LOS}}+ \\
\sqrt{\frac{1}{1+K}}\left(\mathbf{1}_{N_{r} \times N_{t}} \otimes \boldsymbol{\Gamma}\right) \odot\left(\mathbf{\Psi}_{r}^{\frac{1}{2}} \mathbf{H}_{\mathrm{NLOS}} \boldsymbol{\Psi}_{t}^{\frac{1}{2}}\right)
\end{array}
$$

where $\Gamma$ is the leakage matrix

$$
\boldsymbol{\Gamma}=\left[\begin{array}{cc}
\sqrt{1-\mu} & \sqrt{\mu} \\
\sqrt{\mu} & \sqrt{1-\mu}
\end{array}\right]
$$

The operator $\odot$ denotes the element-by-element Hadamard multiplication, $\otimes$ denotes the Kronecker product, $\mathbf{1}_{N_{r} \times N_{t}}$ is an all one $N_{r} \times N_{t}$ matrix, $K$ is the Rician factor, $\mathbf{H}_{\mathrm{LoS}}$ and $\mathbf{H}_{\mathrm{NLoS}}$ are the $2 N_{r} \times 2 N_{t}$ line of sight (LoS) and the non LoS channel matrix respectively. The elements of $\mathbf{H}_{\mathrm{LoS}}$ are deterministic and we will assume that the elements are all one, and $\mathbf{H}_{\mathrm{NLoS}}$ is considered as Rayleigh channel whose elements are independent and identically distributed (iid) circularly symmetric zero mean complex Gaussian variables with unit variance denoted $\mathcal{C N}(0,1)$. Finally, the matrices $\boldsymbol{\Psi}_{r}=\boldsymbol{\Sigma}_{r} \otimes \boldsymbol{\Pi}_{r}$ and $\boldsymbol{\Psi}_{t}=\boldsymbol{\Sigma}_{t} \otimes \boldsymbol{\Pi}_{t}$ are the $2 N_{r} \times 2 N_{r}$ and $2 N_{t} \times 2 N_{t}$ composite transmit and receive correlation matrices, combing spatial and polarization correlations.

\section{PERFormance ANALYSis}

In this section, we evaluate the proposed DP-GSM system in terms of BER (bit-error-rate) and different values of signal-tonoise ratio (SNR) using Monte Carlo simulations over correlated/uncorrelated Rayleigh and Rician channel with different values of $\beta, \mu$ and different amplitude and phase modulation (APM): QPSK, DPSK and 16QAM. For QPSK and DPSK modulations, we choose the configuration $\left(N_{t}=8, N_{a}=4\right.$, $\left.N_{r}=4\right)$. For 16QAM, it is $\left(N_{t}=5, N_{a}=3, N_{r}=4\right)$. The SE for all these systems with QPSK, DPSK and 16QAM is in the order of $18 \mathrm{bpcu}$. Moreover, the corresponding data rate is estimated with a total system bandwidths of $50 \mathrm{GHz}$ which is available in the band between $90 \mathrm{GHz}$ and $200 \mathrm{GHz}$. The current configuration for DP-GSM systems can reach up to $750 \mathrm{Gbps}(\sim 0.75 \mathrm{Tbps})$ for (D)QPSK and 16QAM when the considered total system bandwidth, after channel aggregation and bounding is $50 \mathrm{GHz}$ with 0.2 rolloff factor for the pulse shaping. The simulation parameters, the data rates estimation using DP-GSM is summarized in table I. In all our simulations, the Rician factor $K$ is set to 3 and the polarization
TABLE I

SIMULATION PARAMETERS AND A SUB-THZ SYSTEM EXAMPLE.

\begin{tabular}{|c|c|}
\hline APM & DP-GSM configuration \\
\hline QPSK & $N_{t}=8, N_{a}=4, N_{r}=4$ \\
DQPSK & $N_{t}=8, N_{a}=4, N_{r}=4$ \\
16QAM & $N_{t}=5, N_{a}=3, N_{r}=4$ \\
\hline Parameters for sub-THz & Value \\
System example & 150 \\
\hline Carrier frequency (GHz) & 0.50 \\
Channel bandwidth (GHz) & 18 \\
Spectral Efficiency (bpcu) & 15 \\
Pulse Shaping: Rolloff & 7.5 \\
Spectral efficiency (b/s/Hz) & 750 \\
Data Rates per Channel (Gbps) & 15 \\
Data Rates with 50 GHz (Gbps) & Root Raise cosine:0.2 \\
\hline
\end{tabular}

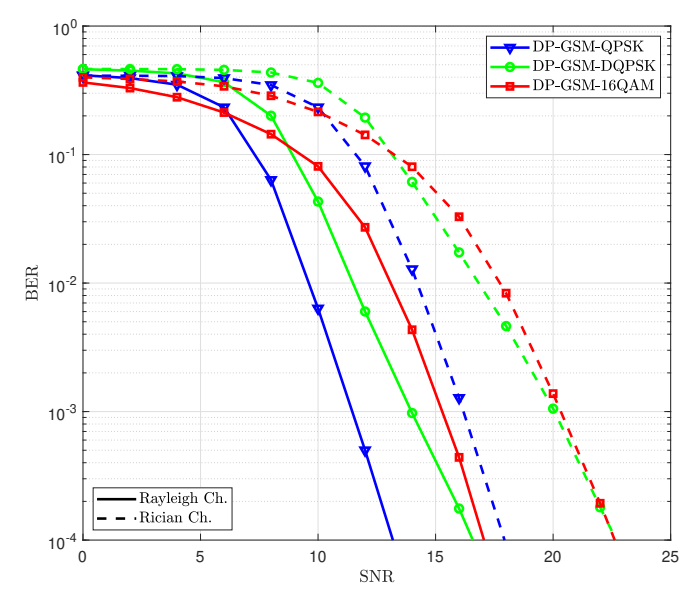

Fig. 2. BER performance of $N_{r} \times N_{t}$ DP-GSM system with power leakage parameter $\mu=0.2$ and different APMs (QPSK, DPSK, 16QAM) over uncorrelated Rayleigh channel (Solid line) and uncorrelated Rician channel (Dashed line). The spectral efficiency is $18 \mathrm{bpcu}$.

correlation for the transmit and the receive components are chosen identical $\left|\gamma_{t}\right|=\left|\gamma_{r}\right|=0.1$.

In figure 2, the performance of different APMs are evaluated over uncorrelated Rayleigh and uncorrelated Rician channel. As it can be shown, the QPSK outperforms the other modulations followed by DQPSK for both cases Rayleigh and Rician channel. It is worth noticing that uncorrelated Rayleigh fading channel gives better performance than in the uncorrelated Rician fading channel. So, for the same BER $\left(10^{-4}\right)$ the required SNR in Rician channel is higher (about 4-5 $d B$ ) than that in Rayleigh channel. In figure 3, the performance of different APMs are evaluated over correlated Rayleigh channel (see Fig 3.(a)) and correlated Rician channel (see Fig 3.(b)) with different correlation levels $\beta=\{0.2,0.5,0.8\}$. The parameter $\mu$ is fixed to 0.2 . The results show that we have a good performance with low spatial correlation level and the performance degrades as $\beta$ value increases. With Rayleigh channel, the performances are much better than in Rician channel. For both channels, we have the same behavior as Fig. 2 which is that QPSK performance with DP-GSM system outperform all the others simulated modulations similar to the conclusion drawn with UP-GSM systems in sub-THz environment [17].

In figure 4, the QPSK modulation is analysed for both Rayleigh (see Fig 4.(a)) and Rician channel (see Fig 4.(b)) 


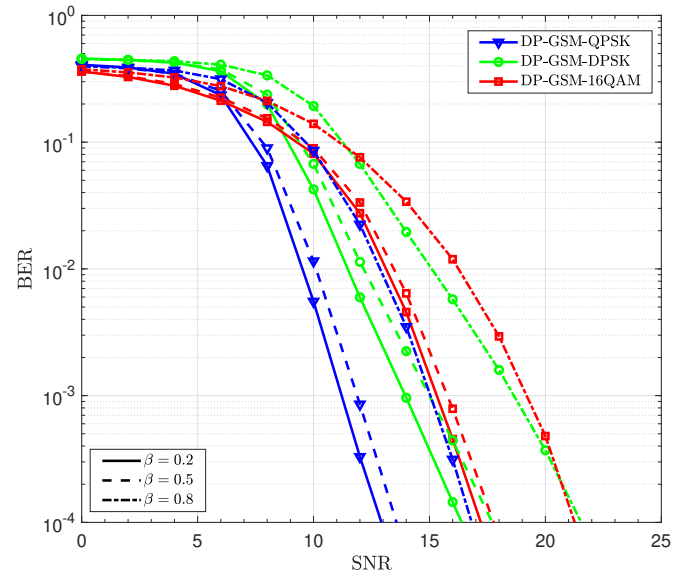

(a)

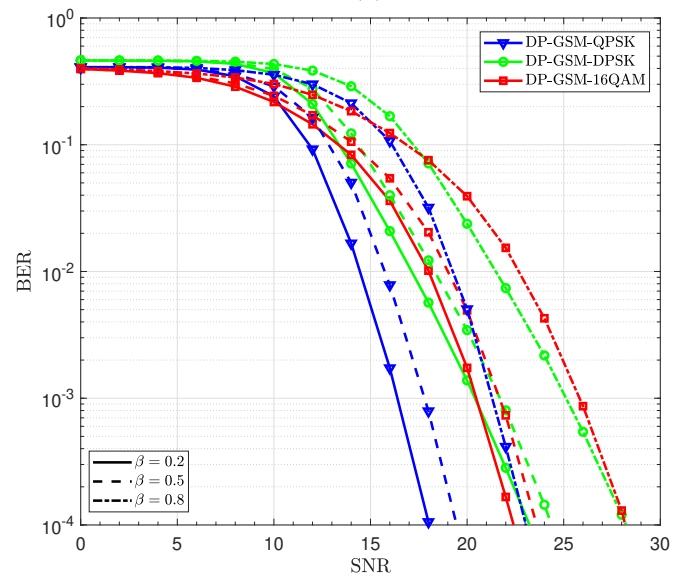

(b)

Fig. 3. BER performance of $N_{r} \times N_{t}$ DP-GSM system with spatial correlation $\beta \in\{0.2,0.5,0.8\}$, power leakage parameter $\mu=0.2$ and different APMs (QPSK, DPSK, 16QAM) over: (a) correlated Rayleigh channel and (b) correlated Rician channel $(K=3)$. The spectral efficiency is $18 \mathrm{bpcu}$.

and the BER performance are plotted as function of the SNR for different configurations of $\beta \in\{0.2,0.5,0.8\}$ and $\mu \in\{0.2,0.8\}$. It can be seen that the DP-GSM system always performs well for low value of $(\mu, \beta)$ and less efficient mainly for a higher spatial correlation $\beta$ value. It is also shown that DP-GSM system have limited degradation $(<1 \mathrm{~dB})$ due to cross-polarization in both channels.

To further illustrate the potential of DP-GSM with the MOB-MMSE detector, the ML detector is compared with our approach. Figure 5 shows the simulation results of the DPGSM system over correlated Rayleigh channel (solid lines) and correlated Rician channel (dashed lines) for QPSK with MOBMMSE and ML detectors. The spatial correlation is chosen $\beta=\{0.2,0.8\}$ and $\mu=0.2$. As it is shown in Fig. 5 there is quite acceptable difference of $(1-2.5) \mathrm{dB}$ between ML and MOB-MMSE at the BER of $10^{-4}$. Thus, the MOB-MMSE for DP-GSM system can achieve near-ML performance with low complexity.

Finally, the DP-SMX and the UP-GSM are compared with the DP-GSM system. We recall the reader that the SMX is a particular case of the GSM technique where symbols

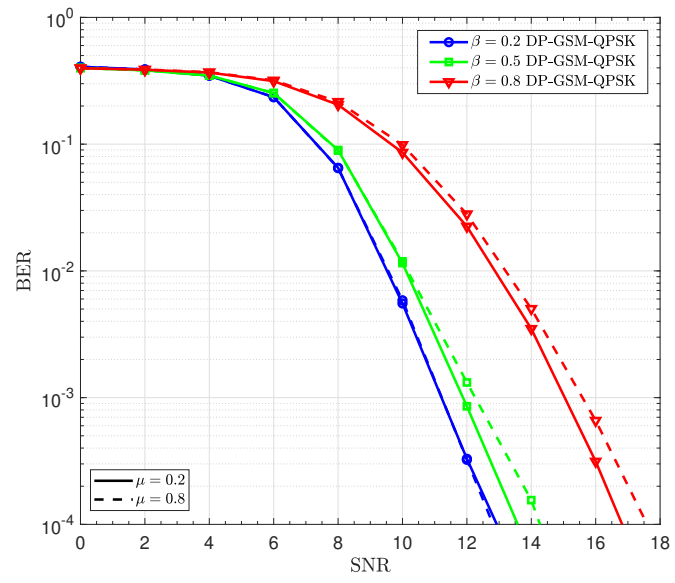

(a)

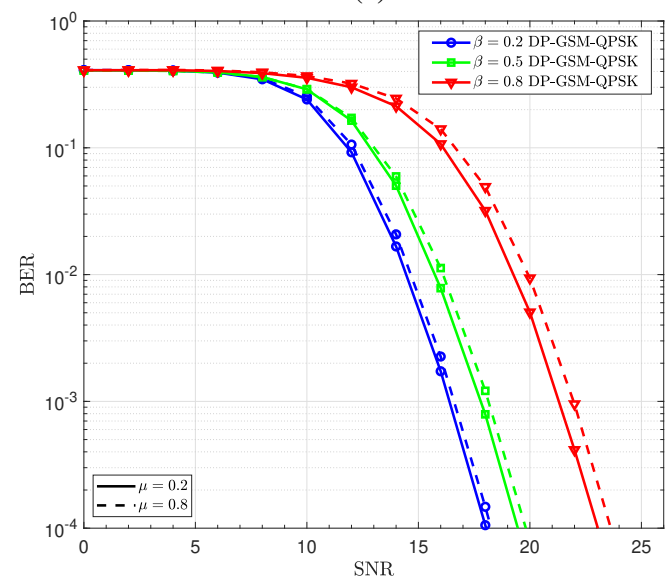

(b)

Fig. 4. BER performance of $N_{r} \times N_{t}$ DP-GSM system with spatial correlation $\beta \in\{0.2,0.5,0.8\}$ and power leakage parameter $\mu=\{0.2,0.8\}$ for QPSK over: (a) correlated Rayleigh channel and (b) correlated Rician channel $(K=$ $3)$. The spectral efficiency is $18 \mathrm{bpcu}$ for $N_{t}=8, N_{a}=4$ and $N_{r}=4$.

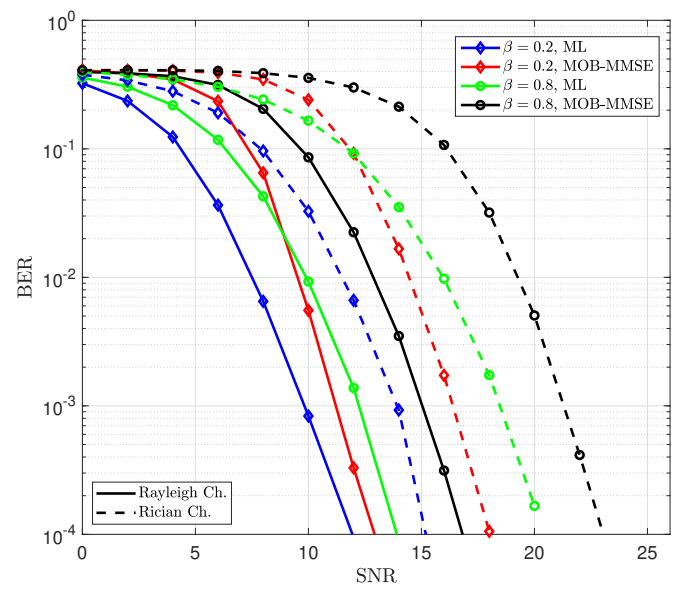

Fig. 5. BER performance comparison of $N_{r} \times N_{t}$ DP-GSM system with spatial correlation $\beta \in\{0.2,0.5,0.8\}$ and power leakage parameter $\mu=$ 0.2 for QPSK over correlated Rayleigh channel (Solid line) and correlated Rician channel (Dashed line) using for both channels MOB-MMSE and ML detectors. The spectral efficiency is $18 \mathrm{bpcu}$.

are transmitted simultaneously from $N_{t}$ TAs to $N_{r}$ receive antennas and all the TAs are activated (here $N_{a}=N_{t}$ ). Note 


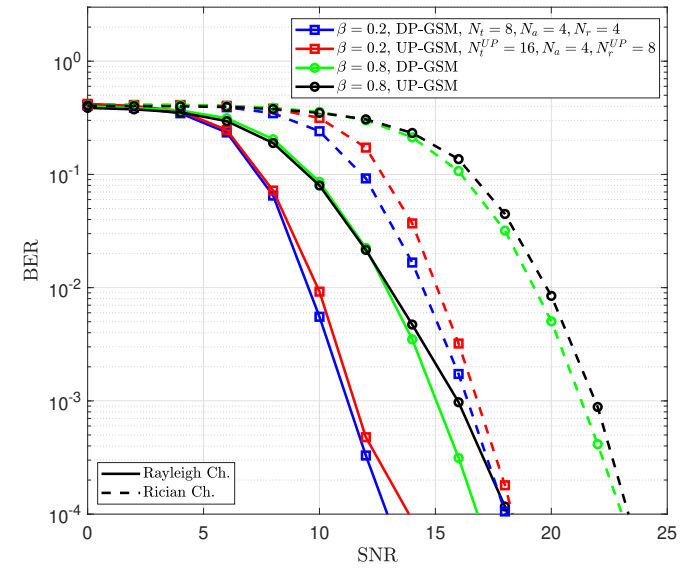

Fig. 6. BER performance comparison of $N_{r} \times N_{t}$ DP-GSM and $2 N_{r} \times 2 N_{t}$ UP-GSM system with spatial correlation $\beta \in\{0.2,0.8\}$ and power leakage $\mu=0.2$ over correlated Rayleigh channel (Solid line) and correlated Rician channel (Dashed line) for QPSK using MOB-MMSE detector. The spectral efficiency is $18 \mathrm{bpcu}$.

that DP-GSM is compared to the best uni-polarized candidate mainly UP-GSM with QPSK and not to SMX, because it was shown in [19] that UP-GSM with QPSK in sub-THz channels has better performance, lower power consumption and detection complexity, and smaller transmitter/receiver antenna array to attain same SE compared to SMX with QPSK. In the DP-SMX system, all transmit and receive antennas use dual polarization to convey simultaneously different symbols on each polarization. The comparison is made using different spatial correlation configurations, $\beta \in\{0.2,0.8\}$, and under different fading channels, Rayleigh and Rician channel. The first comparison is made between the DP-GSM and the UPGSM. The number of antennas for UP-GSM and DP-GSM are $N_{t}^{U P}=16, N_{a}=4, N_{r}^{U P}=8$ and $N_{t}=8, N_{a}=4, N_{r}=4$, respectively, to have same SE given by $18 \mathrm{bpcu}$ and same number of RF chains. It can be seen in Fig. 6 that the performance of UP-GSM and DP-GSM are close with a small SNR gain for DP-GSM. In all cases, the DP-GSM allows to have a more compact antenna array physical size compared to UP-GSM, which is crucial for UE. The second comparison is made between the DP-GSM and DP-SMX using the optimal ML detector where the number of antennas for both systems are respectively, $N_{t}=5, N_{a}=3, N_{r}=3$ and $N_{t}=3, N_{r}=3$, to have same SE given by $12 \mathrm{bpcu}$ and same number of activated TAs for a fair comparison. Figure 7 shows clearly that the DP-GSM and the DP-SMX have the same performance with the ML detector in low spatial correlation and for both fading channels. In the context of high spatial correlation, the DP-GSM outperforms the DP-SMX in both channels and the SNR difference between them is about $1-1.5$ $\mathrm{dB}$ at a BER of $10^{-4}$.

\section{A. Performance with real sub-THz MIMO Channel}

In this work we extended our analysis using a more realistic channel different from that presented in (15). In hereafter we compare the performance of the DP-SMX and the UP-GSM systems using a ray-based deterministic channel modelling for

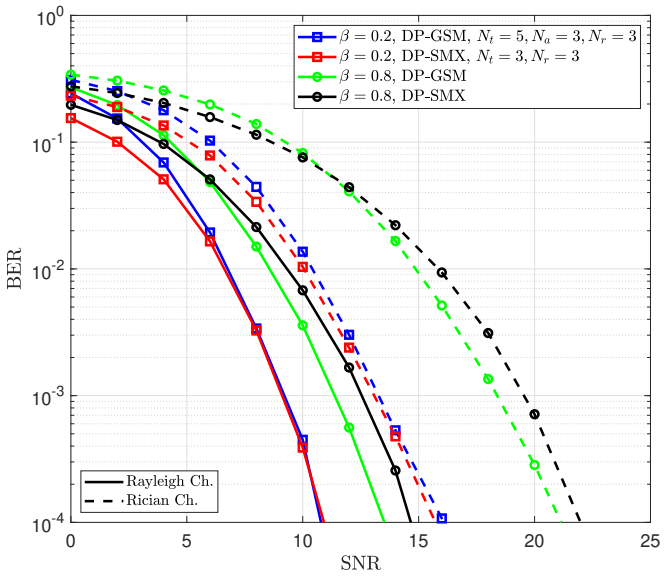

Fig. 7. BER performance comparison of DP-GSM and DP-SMX systems with spatial correlation $\beta \in\{0.2,0.8\}$ and power leakage $\mu=0.2$ over correlated Rayleigh channel (Solid line) and correlated Rician channel (Dashed line) for QPSK using ML detector. The spectral efficiency is $12 \mathrm{bpcu}$.

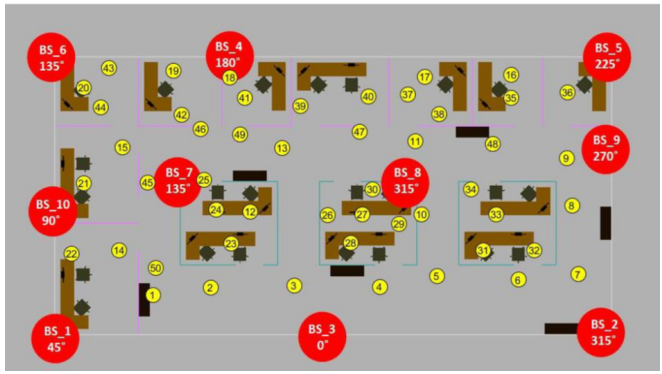

Fig. 8. In-door MIMO channel measurements [18].

sub-THz band (90-200 GHz) developed in [18]. In this channel model, the propagation takes into account several elements such as: material properties, gas attenuation, more propagation paths and new scattered paths. In addition, it characterizes the main channel properties such as path loss and delay spread for LOS, NLOS with vegetation and NLOS cases for indoor in-office and outdoor in-street scenarios. In this subsection, we adopt the downlink hotspot (or kiosk) indoor scenario where the Base Stations (BS), acting as transmitters, and the User Equipment (UE), acting as receivers, are equipped with $N_{t}$ and $N_{r}$ DP antennas. Figure 8 provides a description of a scenario where the $10 \mathrm{BSs}$ are marked in red and distributed throughout the room, and the 50 UEs are highlighted with yellow circles. The corresponding MIMO channels are obtained using the ray-based deterministic channel modeling simulator. Here, Uniform-Linear-Array (ULA) is considered with antenna element separation of $4 \lambda$ for a lower spatial correlation, where $\lambda$ is the wavelength. The considered range for UE is going from $2 \mathrm{~m}$ to $8 \mathrm{~m}$, i.e., we use in average a $5 \mathrm{~m}$ separation distance between UE and BS.

For a fair comparison, the DP-SMX and the UP-GSM are compared with the DP-GSM system under the same SE which is $12 \mathrm{bpcu}$ using same detector, same number of activated TA and number of RF chains. The following configurations are adopted: for DP-GSM ( $N_{t}=5, N_{a}=3, N_{r}=3$ ), for DPSMX $\left(N_{t}=3, N_{r}=3\right)$ and UP-GSM $\left(N_{t}^{U P}=10, N_{a}=3\right.$, 
$N_{r}^{U P}=6$ ). Figure 9, shows such a comparison using the ML detector for the three systems. It can be seen that the DPGSM and DP-SMX have nearly the same performance and they outperform the UP-GSM with a difference of $1.25 \mathrm{~dB}$ at the BER of $10^{-4}$ (see solid lines). Moreover, when the number of DP receive antennas increases: $N_{r}=5$ for both DP-GSM and DP-SMX, and $N_{r}^{U P}=10$ for UP-GSM, the performances of the three systems improve and the DP-GSM and DP-SMX systems always keep their superiority compared to UP-GSM.

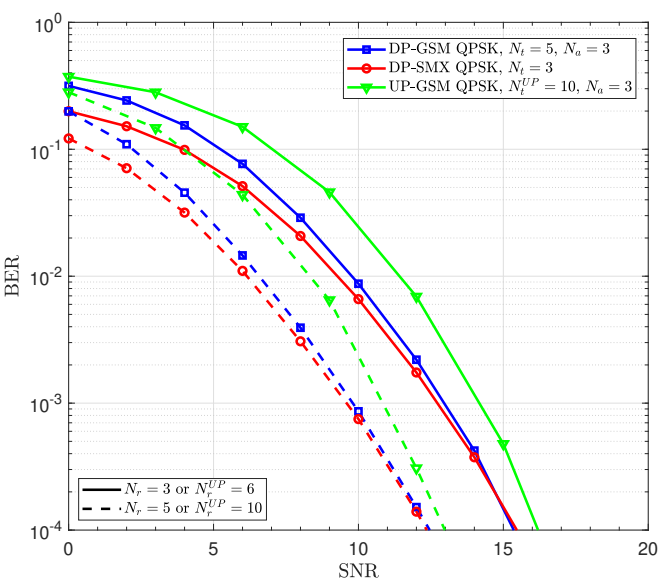

Fig. 9. BER performance of DP-GSM, DP-SMX and UP-GSM systems from sub- $\mathrm{THz}$ measurements for QPSK using ML detector. The concerned sub$\mathrm{THz}$ indoor MIMO channels are generated using ULA array geometry with $4 \lambda$ antenna separation. The spectral efficiency is $12 \mathrm{bpcu}$.

\section{CONCLUSiON}

In this paper, the deployment of the dual polarized antennas leads to the increase of the SE. Therefore, a new polarization dimension is added in the form of $N_{a} \log _{2}\left(N_{p}\right)$. For the detection of the activated antennas indices, their polarization and the transmitted complex symbols for DP-GSM system, a modified OB-MMSE is proposed and applied for the signal detection. In addition, the combination of GSM with DP antennas is evaluated over uncorrelated/correlated Rayleigh and Rician channels. The DP-GSM system offer the opportunities to be more robust against channel deficiency such as Rician fading and inter-antenna correlation, and to be beneficial for improving the space and the SE. The results of comparison with the DP-SMX and UP-GSM systems using both theoretical and sub-THz deterministic fading channel model reveal that DP-GSM has a performance close to the other systems for low spatial correlation. However, it outperforms them at high spatial correlations. The DP-GSM is a promising candidate for ultra-high wireless data rates communication in sub- $\mathrm{THz}$ band.

\section{ACKNOWLEDGMENT}

The research leading to these results received funding from the French National Research Agency (ANR-17-ce25-0013) within the frame of the project BRAVE (Back to single-CaRrier for beyond-5G communications AboVE 90GHz).We would like to thank BRAVE project partner SIRADEL for providing the sub-THz MIMO channels from their ray-based channel simulation.

\section{REFERENCES}

[1] S. Sugiura, S. Chen, and L. Hanzo, "A universal space-time architecture for multiple-antenna aided systems," IEEE Commun. Surv. Tuts., vol. 14,no. 2, pp. 401-420, Jan. 2012.

[2] L. Zheng and D. N. C. Tse, "Diversity and multiplexing: A fundamental trade-off in multiple-antenna channels," IEEE Trans. Inf. Theory, vol. 49,no. 5, pp. 1073-1096, May 2003.

[3] M. Saad, F. Bader, J. Palicot, Y. Corre, G. Gougeon, J-B Dore, "Beyond5G wireless Tbps Scenarios and Requirements," French funded projectANR-17-CE25-0013 BRAVE, Tech. Report BRAVE D1.0, 2018. [Online]. Available: https://hal.archives-ouvertes.fr/hal-01947363/document.

[4] French funded project-ANR-17-CE25-0013, "Back to Single-carrier for beyond-5G communications above $90 \mathrm{GHz}-(\mathrm{BRAVE}), "$ [Online] Available: http://www.brave-beyond5g.com/.

[5] J.-B. Dore, Y. Corre, S. Bicais, J. Palicot, E. Faussurier, D. Ktenas, and F. Bader, "Above-90GHz Spectrum and Single-Carrier Waveform as Enablers for Efficient Tbit/s Wireless Communications," IEEE 25th International Conference on Telecommunications (ICT), 2018.

[6] P. Yang, M. Di Renzo, Y. Xiao, S. Li, and L. Hanzo, "Design guide-lines for spatial modulation," IEEE Commun. Surv. Tuts., vol. 17, no. 1,pp. 6-26, May 2014.

[7] J. T. Wang, S. Y. Jia, J. Song, "Generalised spatial modulation system with multiple active transmit antennas and low complexity detection scheme," IEEE Trans. Wireless Commun., vol.11, no. 4, pp. 1605-1615, Apr. 2012.

[8] M. Di Renzo, H. Haas, A. Ghrayeb, S. Sugiura, and L. Hanzo, "Spatial modulation for generalized MIMO: Challenges, opportunities, and implementation," Proc. IEEE, vol. 102, no. 1, pp. 56-103, Jan. 2014.

[9] M. Saad, F. Bader, J. Palicot, A. C. A. Ghouwayel and H. Hijazi, "Single Carrier with Index Modulation for Low Power Terabit Systems," 2019 IEEE Wireless Communications and Networking Conference (WCNC), Marrakesh, Morocco, 2019, pp. 1-7.

[10] M. Saad, F. C. Lteif, A. C. Al Ghouwayel, H. Hijazi, J. Palicot and F. Bader, "Generalized Spatial Modulation in Highly Correlated Channels," 2019 IEEE 30th International Symposium on Personal, Indoor and Mobile Radio Communications (PIMRC Workshops), Istanbul, Turkey, 2019, pp. 1-6.

[11] G. Zafari, M. Koca and H. Sari, "Dual-Polarized Spatial Modulation Over Correlated Fading Channels," in IEEE Transactions on Communications, vol. 65, no. 3, pp. 1336-1352, March 2017.

[12] Y. Xiao, Z. Yang, L. Dan, P. Yang, L. Yin and W. Xiang, "LowComplexity Signal Detection for Generalized Spatial Modulation," in IEEE Communications Letters, vol. 18, no. 3, pp. 403-406, March 2014.

[13] S. L. Loyka, "Channel capacity of MIMO architecture using the exponential correlation matrix," IEEE Commun. Lett., vol. 5, pp.369-371, Sept. 2001

[14] K. P. Liolis, J. Gomez-Vilardebo, E. Casini and A. I. Perez-Neira, "Statistical Modeling of Dual-Polarized MIMO Land Mobile Satellite Channels," in IEEE Transactions on Communications, vol. 58, no. 11, pp. 3077-3083, November 2010.

[15] C. Oestges, B. Clerckx, M. Guillaud and M. Debbah, "Dual-polarized wireless communications: from propagation models to system performance evaluation," in IEEE Transactions on Wireless Communications, vol. 7, no. 10, pp. 4019-4031, October 2008.

[16] H. Asplund, J. Berg, F. Harrysson, J. Medbo and M. Riback, "Propagation Characteristics of Polarized Radio Waves in Cellular Communications," 2007 IEEE 66th Vehicular Technology Conference, Baltimore, MD, 2007, pp. 839-843.

[17] M. Saad, F. Bader, A. C. Al Ghouwayel, H. Hijazi, N. Bouhel and J. Palicot, "Generalized Spatial Modulation for Wireless Terabits Systems Under Sub-THZ Channel With RF Impairments," ICASSP 2020 2020 IEEE International Conference on Acoustics, Speech and Signal Processing (ICASSP), Barcelona, Spain, 2020, pp. 5135-5139.

[18] G. Gougeon, Y. Corre and M. Z. Aslam, "Ray-based Deterministic Channel Modelling for sub-THz Band," 2019 IEEE 30th International Symposium on Personal, Indoor and Mobile Radio Communications (PIMRC Workshops), Istanbul, Turkey, 2019, pp. 1-6.

[19] M. Saad, A. C. Al Ghouwayel, H. Hijazi, F. Bader and J. Palicot,"MIMO Techniques for Wireless Terabits Systems under sub-THz Channel with RF Impairments," in IEEE International Conference on Communications-ICC, 2020. 